ISSN: 2379-2922

Volume 4, Issue 1, 15 Pages

\title{
Workplace Violence, Anxiety and Self-Esteem in Nursing Staff of Primary, Emergency and Intensive Care Units on the Island of Crete
}

\section{Maria Maniou $^{1 *}$, Sofia Zyga ${ }^{2}$, Spyros Vliamos ${ }^{3}$, Panagiotis Prezerakos ${ }^{4}$, Katerina Flora ${ }^{5}$ Andreas Pavlakis ${ }^{6}$}

${ }^{1 * N}$ Nurse - Computer Engineer, MSc, PhD(c), Organization of Social Solidarity and Education. Municipality of Archanes - Asterousia, Faculty of Nursing - Technological Educational Institute of Crete, Iraklion, Greece

${ }^{2}$ Associate Professor, MSc, PhD, Faculty of Nursing, University of Peloponnese, Sparta, Greece

${ }^{3}$ Dean, Full Professor, Neapolis University, Pafos, Cyprus

${ }^{4}$ Associate Professor, MSc, PhD, Faculty of Nursing, University of Peloponnese, Sparta, Greece

${ }^{5}$ Lecturer in Clinical Psychology, Neapolis University, Pafos, Cyprus

${ }^{6}$ Neapolis University, Paphos, Cyprus

Corresponding Author: Maniou Maria, Nursing Department, Technological Educational Institute of Crete, Greece mmaniou@hotmail.com.

Abstract

Introduction: Mobbing in the workplace occurs when someone repeatedly, for a long time is exposed to negative behaviors and may have difficulty in defending himself. In the nursing profession, labor intimidation is common. It is now accepted that anxiety and self-esteem can play a particularly important role in mental and in physical health of nurses.

Purpose: The investigation of self-esteem, the existence of anxiety and the phenomena of workplace mobbing of nursing staff working in Primary Health Care, Intensive Care Unit and in the Emergency Department.

Methodology: An investigation was carried out in the area of Crete between August 2017 and January 2018 in fourteen Health Centers, two Primary National Health Networks, four Emergency Department, eleven Intensive Care Units. The study involved 213 nurses. The Leymann Inventory of Psychological Terror, the State-Trait Anxiety Inventory and the Culture-free Self-esteem Inventories had been used.

Results: The average age of the participants was 41.73 . The $45.5 \%$ of the sample is working in Intensive Care Units, $24.9 \%$ in Emergency Departments and $29.6 \%$ in Primary Care Units. The mean value of trait anxiety was 40.82 , state anxiety 39.03 , and overall anxiety 79.85 for the overall study sample. They were exposed to at least one mobbing behavior in the past 12 months, and for at least once a week, almost daily or daily the $11.3 \%$ These 24 nurses attribute this behavior to competition problems (60.9\%) and jealousy (58.7\%). The most nurses of the total sample $(50.5 \%)$ had a middle self-esteem.

Conclusions: The evaluation of the results shows that the nursing staff of the overall sample of the study experiences mild anxiety symptoms. Mobbing seems to be at high rates. Early recognition of the phenomenon and its management as well as enhancing of the self-esteem should be the best practice of intervention to prevent it.

\section{INTRODUCTION}

Heinz Leymann was the first who defined workplace bullying that has been a part of working life for centuries (Davenport et al. 1999) and denote a specific form of workplace aggression towards employees [1]. According to Leymann the psychologically violent behaviours he saw in workplaces in Sweden were a kind of 'workplace 
terrorism', and determined it as 'a type of psychological terrorism that arises in the form of directed, systematic, unethical communication and competitive behaviour by one or more persons towards one person' [2]. (Leymann 1990).

The following definition of mobbing see widely agreed upon [3],[4],[5]. (Einarsen et al., 2003, Einarsen, 2000; Einarsen and Skogstad, 1996; Leyman, 1993b; Zapf, 1999a) and it is accepted for this study: Bullying at work means; 'harassing, offending, socially excluding someone or negatively affecting someone's work tasks. In order for the label bullying (or mobbing) to be applied to a particular activity, interaction or process it has to occur repeatedly and regularly (e.g. at least once weekly) and over a period of time (e.g. about six months).

Studies that took part in United Kingdom [6] and Finland [7] shown prevalence rates of around 10\%, whereas in the country of Austria reported results ranged from $7.8 \%$ to $26 \%$ [8]. The lack of a standardized definition and methodology applied to measure workplace bullying contribute probably to the difference in prevalence rates between populations, countries, and organizations [9].

Also, a survey conducted in nursing personnel of the Greek Emergency Departments showed that conflicts between colleagues related to harassment are $24 \%$. A research work for the effect of mobbing on the professional life of nurses in seven Hospitals of the 6th Health Region in Greece showed that nurses, men and women (71\%), were victims of moral harassment during the past year and had psychosomatic symptoms (anxiety $54,3 \%$, headaches $52 \%$, atony $41.5 \%$, denial of work $28 \%$, depression $16,3 \%$ e.g. [10]

Violence in the workplace is expressed as anger, harassment, coercion, intimidation and insult [11]. It is mainly manifested by impacts such as cooperative difficulties, reduced resistance to rush and psychological reactions. etc [12]. It is a chain of anti-social behaviors and an intense form of work-induced anxiety, and through this systematic and long-lasting process (systematic, repetitive, durable and progressive), the worker-victim is led to a psychological and labor extermination [13].

Anxiety was defined by Freud as "something felt", "an emotional state that included feelings of consciousness, intensity, nervousness and anxiety accompanied by normal stimulation.'Parallel to and in conjunction with Darwin's evolutionary theory, Freud observed and noted that stress was adaptive to the motivation of behavior that prompted individuals to deal with threatening situations, and that intense anxiety prevailed in most psychiatric disorders [14]. It has also prevailed, the definition of anxiety as: "A normal physical or psychological reaction to external events that cause a strong emotional state to man. This is a long-standing difficulty or a serious personal event that lasts for at least four (4) weeks "[15].

According to Spielberger, transport concern refers to "subjective emotional state characterized by tension and asphyxia". Permanent anxiety refers to "the predisposition of the individual to perceive specific situations as threatening and to react with the anxiety to them".

Spielberger about permanent and temporary anxiety considers that the recruitment of the individual from both internal and external stimuli is considered as threatening, resulting in reactions of temporary anxiety. Sensory and cognitive feedback mechanisms are the ones that cause high levels of transient stress to be recognized as unpleasant by the individual. The reaction of transient anxiety is proportional to the magnitude of the intensity of the threat.

The sensory and cognitive mechanisms of feedback are those that cause high levels of temporary stress to be recognized as unpleasant by humans. The reaction of temporary anxiety is proportional to the magnitude of the intensity of the threat. Correspondingly, the duration of tension affects the stability of the trait stress response. He also notes that people with a high level of permanent anxiety perceive more situations as threatening and react with more intense transient resistance reactions. Anxiety causes people to develop specific psychological protection mechanisms to achieve the reduction of temporary anxiety [16]. 
Nursing, as a profession, is described as a "high intensity profession", the reasons have already been extensively formulated. This in itself has negative effects on the self-esteem of the nurse, both as a person and as a professional. In Greece, a survey was conducted in three (3) public hospitals in the Prefecture of Ilia during the period 05-10 / 2008, with a sample of 167 nurses and nursing assistants aged 20-60 years old. The statistical analysis of the questionnaire showed that $56.3 \%$ of the sample of nurses has a "modest'emotional state, which affects their self-esteem [17].

There are no surveys in Greece related to the correlation of work harassment with permanent, temporary anxiety and self-esteem in nursing staff. The first aim of this study was to measure the prevalence and forms of workplace bullying among the nursing personnel working in the Primary Health Care, Emergency Departments (ED) and Intensive Care Units (ICU) of Crete. The first approximation comprises the measurement of the frequency and duration of exposure to one or more of the 45 forms of bullying [18] and the second approximation include a self-reporting question revelant to bullying within the past 12 months based on a definition according to Heinz Leymann [19]. The second aim was to measure the existence of state and trait anxiety with two questionnaires [20] and the third aim was to measure the self esteem of the nursing personnel.

\section{General OBJECTIVE}

The general objective of the study was to investigate the state and trait anxiety, the work violence and the self esteem in nursing staff in Primary Health Care, in Emergency Departments (ED) and in Intensive Care Units (ICU) of five major hospitals and 11 Health Centers in the prefecture of Crete.

\section{SPECIFIC ОBJECTIVES}

- To study the existence and to measure the prevalence of anxiety in nursing staff

- To study the existence and to measure the prevalence of work violence in nursing staff

- To measure the self esteem in nursing staff

\section{RESEARCH QUESTIONS}

- The work violence experienced by nursing staff of Intensive Care Units and Emergency Departments differs from that of nursing staff in Primary Health Care.

- The permanent and trait anxiety suffered by nursing staff of Intensive Care Units and Emergency Departments differs from that of nursing staff in Primary Health Care.

- The self-esteem of nursing staff of the Intensive Care Units and Emergency Departments differs from that of nursing staff in Primary Health Care.

\section{SigNifiCANCE OF THE STUDY}

The present study is expected to be used by Human Resource Management of the Hospitals and Health Care Centres to design appropriate policies that can curb mobbing and anxiety.

\section{METHOdOLOGY}

The study involved 213 nurses from five hospitals in the region of Crete. Of these, $45.5 \%$ were working in Intensive Care Units while the other $24.9 \%$ were working at the Emergency Departments and $29.6 \%$ in Primary Health Care. The survey was conducted from August 2017 to January 2018 and included the voluntary and anonymous participation of nursing staff. The psychometric tools included in the study are presented below:

\section{INSTRUMENTS}

For the purpose of the present research, the French version of "Leymann's Inventory of Psychological Terror'(LIPT) instrument [21] and the Greek Version of "Leymann Inventory of Psychological Terror' Instrument were applied [22]. 
Leymann's Inventory of Psychological Terror consists of 45 items, each item measuring the exposure to workplace bullying the last 12 months with two response options (yes or no). In addition, two questions on the frequency were included (monthly basis, weekly, or daily) as well as the duration of bullying (years and months). In five sections are grouped the 45 bullying behaviors (1) social relationships at work (criticism, no possibility to communicate, and indifference and verbal aggression), (2) exclusion (isolation, avoidance and rejection ), (3) job tasks (too many tasks, no tasks, uninteresting tasks, humiliating tasks, tasks superior, or inferior to skills), (4) personal attacks (attacks on origins or opinion, rumors, ridicule and gossiping), and (5) physical violence (physical threats and sexual annoyance). According to Leymann, those who report exposure to at least one of the 45 behaviors that concerns bulling the last 12 months, weekly or more, and for six months or longer are defined as victims of bullying [23].

Moreover, in the present study will be used questions included in the French version of LIPT instrument. The instrument includes the definition of workplace bullying: "Bullying may be defined by a situation in which someone is exposed to a hostile behavior on the part of one or more persons in the work environment that aim continually and repeatedly to offend, oppress, maltreat or to exclude or isolate over a long period of time'[4]. a. Nurses are asked whether they aware themselves as being victims of bulling within the previous year. For those considering themselves as being victims, contributing factors are further researched. b. In addition, nurses are asked whether they witnessed bullying at the current work to another employee during the past 12 months.

The State-Trait Anxiety Inventory (STAI) is a psychological inventory based on a 4-point Likert scale. It consists of 40 questions. The STAI measures two types of anxiety - state anxiety, or anxiety about an event and trait anxiety or anxiety level as a personal characteristic. Higher scores are positively correlated with higher levels of anxiety.

State anxiety (S-anxiety) can be defined as discomfort, fear, nervousness, etc. and the arousal of the autonomic nervous system induced by different situations that are apprehend as dangerous and is considered temporary. Trait anxiety (T-anxiety) can be defined as feelings of worry, stress discomfort, etc. that one experiences and how people feel across typical situation daily. The State-Trait Anxiety Inventory assess both state and trait anxiety separately. Each type of anxiety has its own scale of 20 questions and the scores range from 20 to 80 , with higher scores correlating with higher levels anxiety. Each scale asks twenty questions each and based on a 4-point Likert scale. Low scores show a mild form of anxiety. Median scores indicate a moderate form of anxiety and high scores shows a severe form of anxiety. Anxiety absent questions impersonate the absence of anxiety in a statement like, "I feel secure.'Anxiety declares questions represent the presence of anxiety e.g. "I feel worried.'More examples from the STAI on anxiety absent and present questions are below. The 4-point scale for S-anxiety is as follows: 1.) not at all, 2.) somewhat, 3.) moderately so, 4.) very much so and for the 4-point scale for T-anxiety is: 1.) almost never, 2.) sometimes, 3.) often, 4.) almost always [24].

The Culture-free Self-esteem Inventories is a self-referencing questionnaire, which includes (without the lie scale) 32 statements. These statements seek to measure the general, personal and social perception of themselves (self-perception) and are divided into two groups: those that are high and those showing low self-esteem. Each question answers either with "yes'or with "no". Three self-assessment sub-scales are included: general (16 statements), social (8 statements) and personal (8 statements). There is also a lie scale o (8 statements) [25].

Permissions were obtained from the developers of the French version of LIPT instrument, the Greek version of LIPT instrument, the State-Trait Anxiety Inventory (STAI) and Culture-free Self-esteem Inventories. The time needed to fill out the questionnaire was 8-10 minutes.

\section{STUdy POPUlation}

The present study conducted among nurses working in primary health-care setting with the participation of 14 Health-care Centers and 5 Hospitals in the island of Crete. A total of 213 nurses take part in the study. The study 
was performed in the following departments: 14 Health-care Centers, 4 Emergency Departments (ED) and 11 Intensive Care Units (ICU). The study was performed during August 2017 to January 2018.

\section{Inclusion Criteria}

- Selected participants had to be nursing staff

- Selected participants had to be staff coming from all Intensive Care Units, Emergency Departments and Primary Health Care Centres of Crete

- Selected participants had to work to Hospitals and Health Centers that had been included in the National Health System and had the same system of shift to ensure the homogeneity of the sample.

- Selected participants could have any educational level with each working relationship

\section{Exclusion Criteria}

- Participants had not to be nursing student

\section{Statistical Analysis}

With respect to the statistical analysis that follows, the quantitative variables are reported based on the mean \pm standard deviation (mean $\pm \mathrm{sd}$ ) as well as the median and the interquartile range (IQR), while for the qualitative variables we have the corresponding frequencies and percentages. Depending on the appropriate statistical and / or graphic controls, it is recommended that median and the interquartile range (median, IQR) are used as representative descriptive measures.

The Shapiro-Wilk statistical control was used to check the regularity of the quantitative variables in the questionnaire. The existence of regularity has also been confirmed or rejected by the visual overview of the corresponding histograms, the normal Q-Q plots and box-plots of the variables. At the same time, the appropriate parametric and non-parametric statistical examinations were conducted to investigate any differences between the three structures (ICU, KY / PEDY and TEI) and the scales under study, determining the level of significance at 0.05 . In all cases it was necessary to use accurate tests and / or model-carousel simulation (10000 samples). Also, where necessary, the Levene test was used for the homogeneity of the difference.

Finally, reliability and internal consistency was assessed by internal consistency using Cronbach's alpha test. Statistical analysis was performed using the statistical software IBM SPSS statistics (version 21.0). A p value $<0.05$ was considered statistically significant.

\section{ETHics APPROVAL}

The researcher submitted the research protocol for obtaining the necessary written permissions from the Research and Ethics Committees of the University General Hospital of Heraklion, General Hospital of Heraklion "Venizelio Pananio", General Hospital of Chania, General Hospital of Agios Nikolaos, General Hospital of Rethymno and the 7th Health District of Crete. The permissions of the above organizations were given provided that the results of the study will be announced in the Administration of the 7th Health District of Crete. After the permissions of the research ware given, the participants were approached. Written consent was given by all participants to participate in the study.

\section{RESUlts}

\section{Reliability of Leymann's Inventory of Psychological Terror'(LIPT) Instrument}

The reliability of LIPT instrument expressed by Cronbach $\alpha$ was 0.938 suggesting high internal consistency. 
Workplace Violence, Anxiety and Self-Esteem in Nursing Staff of Primary, Emergency and Intensive Care Units on the Island of Crete

\section{Characteristics of the Study Sample}

In this study, the participants were nursing staff $(n=213)$ and the majority of the participants $89.2 \%$ of the sample were women and ICU nurses represented $45.5 \%$ of the study population. Mean age for the total sample was 41.73 (SD = 7.33 years). The 75.1\% $(\mathrm{n}=160)$ of the sample were married and followed by $23.0 \%(\mathrm{n}=23 \%)$ that were unmarried. The majority of the sample $64.8 \%(n=138)$ were graduates of Technological Educational Institute (TEI) also, $8.9 \%$ of the sample $(\mathrm{n}=18)$ had a master's degree and one person had a PhD. Demographic characteristics of the research population are shown in Table 1. In regard to the mean length of employment was 15.78 ( $\mathrm{SD}=8.49$ years). The average of work in the current department was 8.00 years $(\mathrm{SD}=10.50$ years) as shown in the table below Table 2 .

Table1. Characteristics of the study population $(N=296)$.

\begin{tabular}{|c|c|c|}
\hline \multicolumn{2}{|c|}{ Characteristics } & n (\%) \\
\hline & & $97(45.5)$ \\
\hline Departments & Intensive Care Unit & $63(29.6)$ \\
\hline & Primary Care & $53(24.9)$ \\
\hline & Emergency Department & $23(10.8)$ \\
\hline Gender & Man & $190(89.2)$ \\
\hline Marital Status & Women & $160(75.1)$ \\
\hline & Married & $49(23.0)$ \\
\hline & Unmarried & $4(1.9)$ \\
\hline Educational Level & Widowed/Divorced & $43(20.2)$ \\
\hline & Secondary School & $138(64.8)$ \\
\hline & Technological Educational Institute & $13(6.1)$ \\
\hline & University & $18(8.4)$ \\
\hline & Master degree & $1(0.5)$ \\
\hline
\end{tabular}

Table2. Descriptive characteristics among the study population $(N=213)$.

\begin{tabular}{|c|c|}
\hline 1. Characteristics & \\
\hline Age (years) & $41.73 \pm 7.33^{*}$ \\
\hline Years of work & $15.78 \pm 8.49^{*}$ \\
\hline Years of work in the present department & $8.00(10.50)^{* *}$ \\
\hline
\end{tabular}

$*$ mean $\pm \mathrm{sd}$

**median (IQR)

\section{Prevalence of Workplace Bullying among Nurses}

According to Leymann, mobbing is defined as the worker's exposure at least once a week for the past 12 months. Based on this definition, we have the following results. Among the 213 nurses that consisted the total sample of the study, 95 nurses (44.6\%) were exposed to at least one bullying behavior at work within the last 12 months, whereas 24 nurses (32.43\%) were exposed to at least one bullying behavior or more at least once weekly the last 12 months as shown in the table below Table 3. There is a statistically significant difference between the three structures $(p=0.016)$ in the percentages of those who said they were exposed to at least one mobbing (out of 45) in the last 12 months. We observe that the highest rate of exposure to at least one 
Workplace Violence, Anxiety and Self-Esteem in Nursing Staff of Primary, Emergency and Intensive Care Units on the Island of Crete

moral harassment behavior is reported in the Emergency Department (58.5\%), while the lowest percentage in Primary Care (31.7\%) as shown in the table below Table 4 . With regard to the frequency of the mobbing in the last 12 months and the answers "rarely'or "at least once a month'and "at least once a week'or "almost daily'or "daily", compared to the structures, there is no statistically significant difference, as shown in the table below Table 5. The percentage of the nurses still continuing to experience similar situations is $69.23 \%(n=54)$. The median of the time period for which someone with these conditions had come was 12 months (IQR = 51.75). The prevalence of the victims of workplace bullying among nurses working at the Intensive Care Units was $10(41.7 \%)$ whereas among those working at the Emergency Departments (ED) was 10(41.7\%), and among nurses working at the Primary Health Care was 4(16.6\%).

Table3. Prevalence of exposure to each of the 45 mobbing behaviors among the study population during the last 12 months $(N=213)$.

\begin{tabular}{|c|c|}
\hline & Yes $(\%)$ \\
\hline \multicolumn{2}{|l|}{ Industrial Relations } \\
\hline Being silenced by superior & 8.5 \\
\hline Being continuously interrupted & 10.3 \\
\hline Being silenced by others & 14.6 \\
\hline Being scolded and yelled & 11.3 \\
\hline Being criticized regarding work assignments & 9.4 \\
\hline Private life being criticized by others & 5.2 \\
\hline Being terrorized by means of phone calls & 5.2 \\
\hline Receiving verbal threats & 7.5 \\
\hline Receiving written threats & 2.3 \\
\hline Being exposed to irritating gestures/looks & 14.6 \\
\hline Physical presence ignored, addressing only others & 9.4 \\
\hline \multicolumn{2}{|l|}{ They isolate you systematically } \\
\hline Not being talked to & 5.6 \\
\hline Not being allowed to physically contact others & 3.3 \\
\hline Being isolated from others at work & 3.3 \\
\hline Conversation with colleagues is forbidden & 1.9 \\
\hline Physical presence being ignored among others & 5.2 \\
\hline Being addressed only in written ways & 0.5 \\
\hline \multicolumn{2}{|l|}{ Your professional duties have been amended as a punishment } \\
\hline They do not assign you jobs, you have no job & 0.9 \\
\hline Being given meaningless work assignments & 7.5 \\
\hline Being given work assignments far below capacity & 6.1 \\
\hline Continuously being given new work assignments & 8.5 \\
\hline Being given humiliating work assignments & 2.8 \\
\hline Being given difficult work assignments far above capacity & 7.5 \\
\hline \multicolumn{2}{|l|}{ Attacks on your face } \\
\hline Being gossiped & 15.0 \\
\hline Being exposed to slanders and lies & 12.2 \\
\hline Being ridiculed & 4.7 \\
\hline Being said to have a mental illness & 1.9 \\
\hline Being forced to go through psychiatric exams & 1.9 \\
\hline Being mocked due to a handicap that you have & 4.2 \\
\hline Voice, gestures, and way of moving are imitated to tease & 5.6 \\
\hline Suffering verbal attacks regarding political and religious beliefs & 3.3 \\
\hline
\end{tabular}


Workplace Violence, Anxiety and Self-Esteem in Nursing Staff of Primary, Emergency and Intensive Care Units on the Island of Crete

\begin{tabular}{|c|c|}
\hline Being teased due to ethnic background & 3.8 \\
\hline Being forced to do work assignments which are against your conscious & 8.9 \\
\hline Being judged for your work in an injustice and humiliating way & 11.3 \\
\hline Your decisions are questioned by others & 8.9 \\
\hline Being reviled using obscene or degrading terms & 2.8 \\
\hline Being sexually threaten & 2.3 \\
\hline Violence and threats of violence & 4.7 \\
\hline Being given dangerous work assignments for your health & 7.0 \\
\hline Despite your bad health you are forced to do work assignments that heart your health & 0.9 \\
\hline Being physically threaten & 2.8 \\
\hline Being physically threatened in the form of mild violence as a warning & 1.4 \\
\hline Being physically attacked & 0.5 \\
\hline Being forced to spend big sums of money & 1.9 \\
\hline Workplace or home is damaged by others & 0.0 \\
\hline Being sexually attacked & $13)$ \\
\hline
\end{tabular}

Table4. Mobbing in departments among the study population during the last 12 months ( $N=213)$.

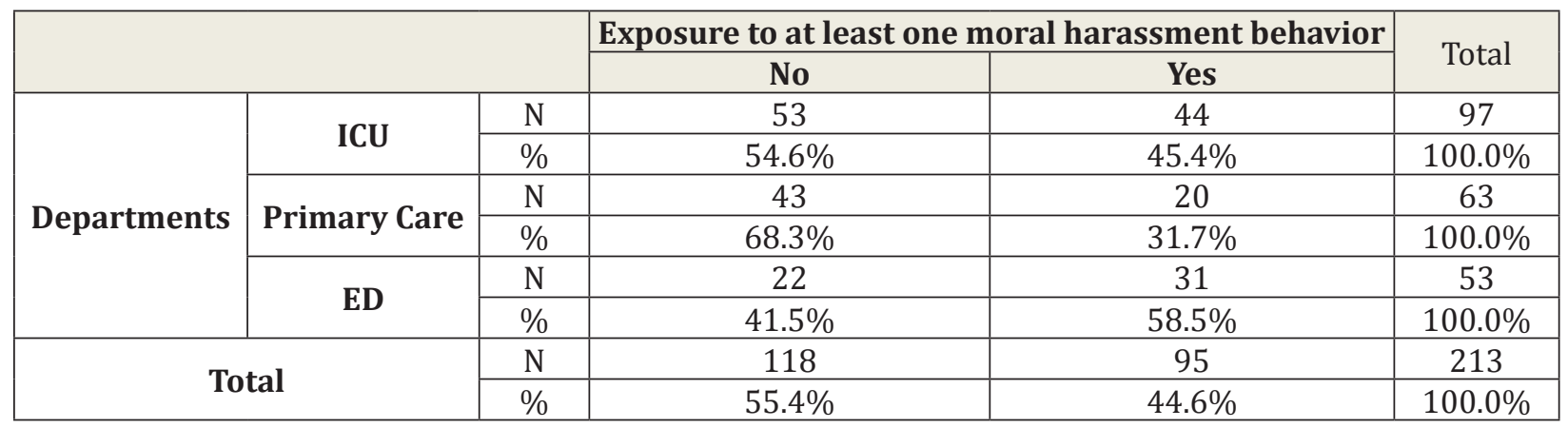

$\chi^{2}(2)=8.374, p=0.016$

Table5. Frequency of mobbing in departments.

\begin{tabular}{|c|c|c|c|c|c|}
\hline & \multicolumn{2}{|c|}{$\begin{array}{l}\text { How often have you been confronted with one or } \\
\text { more of the above situations in the last } 12 \text { months? }\end{array}$} & \multirow{2}{*}{ Total } \\
\hline & & & \begin{tabular}{|c|}
$\begin{array}{c}\text { Rarely / At least once } \\
\text { a month }\end{array}$ \\
\end{tabular} & $\begin{array}{l}\text { At least once a week / } \\
\text { Almost daily / Everyday }\end{array}$ & \\
\hline \multirow{6}{*}{ Departments } & \multirow[b]{2}{*}{ ICU } & $\mathrm{n}$ & 23 & 10 & 33 \\
\hline & & $\%$ & $69.7 \%$ & $30.3 \%$ & $100.0 \%$ \\
\hline & \multirow{2}{*}{ Primary Care } & $\mathrm{n}$ & 12 & 4 & 16 \\
\hline & & $\%$ & $75.0 \%$ & $25.0 \%$ & $100.0 \%$ \\
\hline & \multirow{2}{*}{ ED } & $\mathrm{n}$ & 15 & 10 & 25 \\
\hline & & $\%$ & $60.0 \%$ & $40.0 \%$ & $100.0 \%$ \\
\hline \multirow{2}{*}{\multicolumn{2}{|c|}{ Total }} & $\mathrm{n}$ & 50 & 24 & 74 \\
\hline & & $\%$ & $67.6 \%$ & $32.4 \%$ & $100.0 \%$ \\
\hline
\end{tabular}

$\chi^{2}(2)=1.125, p=0.625$

According to Niedhammer's definition $16.9 \%$ of the total sample reported exposure in bulling behaviors at their workplace the last 12 months. Between them the $16.5 \%, 9.5 \%$ and $26.4 \%$ of nurses working at the ICU, Primary Health Care and Emergency Department respectively. Finally, based on the definition of psychological 
violence at work, there was a statistically significant difference between the three departments $(p=0.047)$, with the highest percentage reported by Emergency Departments (26.4\%).

Moreover colleagues, chiefs or persons holding higher rank in hierarchy were most commonly recognized as mobbers between participants $(43.9 \%, 43.9 \%$ respectively). With regard to the sex of the persons who have practiced psychological violence, $43.31 \%$ were women, $13.58 \%$ were men and $43.31 \%$ were both of them (women and men). The median of the number of people who were against those who received psychological violence during this period was $2(\mathrm{IQR}=2)$.

\section{Bullying Behaviors and Frequency of Exposure to Workplace Bullying}

The most common mobbing behaviors reported among the participants were the following: "being badly criticized behind your back'(15.0\%); with the same percentage appears "being continuously interrupted by others'and "being accepted contemptuous glances and / or contemptuous gestures'(14.6\%); "being exposed to slanders and lies'(12.2\%); and with the same percentage appears "being vilified and shouted'and "being criticized regarding work assignments'(11.3\%).

According to the definition of mobbing as proposed by Niedhammer et al $[13,29.3 \%$ of the study participants answered positively regarding exposure to hostile behaviors within the last 12 months (45.4\% of those working at the ICU, 31.7\% of those working in Primary Care and 58,5 of those working in Emergency Department respectively). There is a statistically significant result $(\mathrm{p}=0.016)$. We observe that the highest rate of exposure to at least one behavior of moral harassment is reported in Emergency Department (58.5\%), while the lowest rate in Primary Care (31.7\%).

When applying the definition of mobbing as proposed by Niedhammer et al [13, 36 nurses (16.9\%) of the study participants answered that having been subjected to psychological violence at work in the last 12 months. $(16, .5 \%$ of those working at the ICU, 9.5\% of those working at the Primary Health Care and $26.4 \%$ of those working at the Emergancy Department, respectively). The most common causes responsible for the exposure to hostile behaviors for the total sample were "competitive behaviors between employees' $(60.9 \%)$ and "jealousy'(58.7\%), followed by "a generally bad working environment' $(17.0 \%)$ and "problems in the management and professional position'(14.2\%). The $30.0 \%$ of the total sample of the individuals reported that they became observers of mobbing behaviors against another employee during the previous year.

\section{Reliability of the State-Trait Anxiety Inventory (STAI)}

The reliability of STAI instrument expressed by Cronbach $\alpha$ was 0.940 suggesting high internal consistency (State anxiety: alpha $=0.895$, Trait anxiety: alpha $=0.907$ ).

\section{Prevalence of State - Trait Anxiety}

The mean value of trait anxiety was 40.82 , the mean value of state anxiety was 39.03 and the total anxiety was 79.85 Table 6

Concerning the first subscale for the emotional state (trait anxiety) of the interviewee at the time of completing the questionnaire we have the following results. Based on ANOVA, there is no statistically significant difference in the mean score of Spielberger 1, between the three structures: F $(2.210)=1.991, p=0.139$, Intensive Care Units (ICU): $41.74 \pm 11.59$, Primary Care: $35.57 \pm 9.62$, Emergency Department (ED): $41.81 \pm 10.04$. Based on the Tukey HSD test for comparisons between two structures, there was no statistically significant difference between ICU-Primary Care $(p=0.160)$, nor between ICU / ED ( $p=p=0.236)$ nor between Primary Care - ED $(\mathrm{p}=0.236)$.

Regarding the second sub-scale for the moral status (state anxiety) of the persons how they feels general in their lives, based on ANOVA, there is no statistically significant difference in the mean score of this unit of the three structures: F $(2.210)=2.752$, p $=0.066$, ICU: $40.28 \pm 10.58$, Primary Care: $36.59 \pm 8.50$, ED: $39.66 \pm$ 
Workplace Violence, Anxiety and Self-Esteem in Nursing Staff of Primary, Emergency and Intensive Care Units on the Island of Crete

10.44. Based on the Tukey HSD for comparisons between two structures, there was no statistically significant difference between the ICU-Primary Care $(p=0.060)$, neither between the ICU-ED $(p=0.930)$ nor between the Primary Care-ED $(\mathrm{p}=0.226)$.

Regarding Spielberger's overall scale and overall score, we have the following results. First of all, the homogeneity of the dispersion did not apply, so we proceeded to the Welch test, which resulted in a statistically significant difference between the three groups, $p=0.039$, ICU: $82.02 \pm 21.16$, Primary Care: $75.16 \pm 15.53,19.22$. However, based on the two-time comparisons and on the basis of the Tamhane and Dunett T3 controls, there was no statistically significant result between the ICU-Primary Care $(p=0.057)$, nor between the ICU-ED $(p=0.998)$ between Primary Care-ED ( $\mathrm{p}=0.162)$.

\section{Reliability of Culture-free Self-Esteem Inventories (James Battle)}

The reliability of Culture-free Self-esteem Inventories (James Battle) expressed by Cronbach $\alpha$ was 0.763 suggesting high internal consistency (General self-esteem: alpha $=0.737$, Social self-esteem: alpha $=0.442$, Personal self-esteem: alpha $=0.724$, Lie scale: alpha $=0.525$, General self-esteem: alpha $=0.763$ ).

\section{Valuation of Self-Esteem}

From the analysis of the results of the Culture-free Self-esteem Inventories (James Battle), it was found that $51.6 \%$ of the nurses who participated in the study had a middle self-esteem, while the lowest percentage of the total sample of $8.0 \%$ showed very high self-esteem. Also, it was found that $8.0 \%$ had very low self-esteem, $17.4 \%$ had low self-esteem and $15 \%$ had high self-esteem. Also, the results showed that the General self-esteem was 12.27 (mean), the Social self-esteem 6.35(mean), the Personal self-esteem was 3.86 (mean) and the scale of lie was 4.77 Table 6. Regarding the Culture-free Self-esteem Inventories and based on the chi-square test was applied. There are no statistically significant differences between the three structures (ICU, ED, Primary Care) compared to the level of self-esteem, despite the fairly large difference in many percentages. Something that is probably due to the existence of many categories at the level of self-esteem. With regard to the level of selfesteem for the three structures (ICU, ED, Primary Care) we had the following results: ICU: very low self-esteem: (8.2\%), low self-esteem (17.5\%), middle self-esteem (50.5\%), high self-esteem (13.4\%), very high self-esteem (10.3\%), ED: very low self-esteem: (11.3\%), low self-esteem (26.4\%), middle self-esteem (37.7\%), high selfesteem (20.8\%), very high self-esteem (3.8\%), Primary Care: very low self-esteem: $(4.8 \%)$, low self-esteem (9.5\%), middle self-esteem (65.1\%), high self-esteem (12.7\%), very high self-esteem (7.9\%) Table 7.

Table6. Descriptive characteristics of the scales among the study population ( $N=213)$.

\begin{tabular}{|c|c|c|c|c|c|c|}
\hline Scales & n (\%) & Mean & SD & Median & IQR & Range \\
\hline State-Trait Anxiety Inventory (STAI) & & & & & & \\
\hline STAI - Part 1 & & 40.82 & 10.72 & 39.00 & 16.00 & $23-68$ \\
\hline STAI - Part 2 & & 39.03 & 10.06 & 38.00 & 13.50 & $22-70$ \\
\hline STAI - (Part 1 \& Part 2) & & 79.85 & 19.32 & 77.00 & 25.00 & $47-138$ \\
\hline Culture-free Self-esteem Inventories (James Battle), & & & & & & \\
\hline General self-esteem * & & 12.27 & 2.89 & 13.00 & 3.50 & $2-16$ \\
\hline Social self-esteem * & & 6.35 & 1.38 & 7.00 & 1.00 & $1-8$ \\
\hline Personal self-esteem * & & 4.86 & 2.16 & 4.00 & 3.50 & $0-8$ \\
\hline Scale of lie & & 22.47 & 1.56 & 5.00 & 2.00 & $0-8$ \\
\hline Total self-esteem & $17(8.0)$ & & & & & \\
\hline Too low & $37(17.4)$ & & & & & \\
\hline Low & $110(51.6)$ & & & & & \\
\hline Middle & $32(15.0)$ & & & & & \\
\hline Hugh & $17(8.0)$ & & & & & \\
\hline Very Hugh & & & &
\end{tabular}

*(IQRs) as representative descriptive measures for these scales 
Workplace Violence, Anxiety and Self-Esteem in Nursing Staff of Primary, Emergency and Intensive Care Units on the Island of Crete

Table7. Level of self-esteem among the study population $(N=213)$.

\begin{tabular}{|c|c|c|c|c|c|c|c|c|}
\hline & \multicolumn{5}{|c|}{ Level of self-esteem } & \multirow{2}{*}{ Total } \\
\hline & & & Very Low & Low & Middle & High & Very high & \\
\hline \multirow{6}{*}{ Department } & \multirow{2}{*}{ ICU } & $\mathrm{N}$ & 8 & 17 & 49 & 13 & 10 & 97 \\
\hline & & $\%$ & $8.2 \%$ & $17.5 \%$ & $50.5 \%$ & $13.4 \%$ & $10.3 \%$ & $100.0 \%$ \\
\hline & \multirow{2}{*}{ Primary Care } & $\mathrm{N}$ & 3 & 6 & 41 & 8 & 5 & 63 \\
\hline & & $\%$ & $4.8 \%$ & $9.5 \%$ & $65.1 \%$ & $12.7 \%$ & $7.9 \%$ & $100.0 \%$ \\
\hline & \multirow{2}{*}{ ED } & $\mathrm{N}$ & 6 & 14 & 20 & 11 & 2 & 53 \\
\hline & & $\%$ & $11.3 \%$ & $26.4 \%$ & $37.7 \%$ & $20.8 \%$ & $3.8 \%$ & $100.0 \%$ \\
\hline \multirow{2}{*}{\multicolumn{2}{|c|}{ Total }} & $\mathrm{N}$ & 17 & 37 & 110 & 32 & 17 & 213 \\
\hline & & $\%$ & $8.0 \%$ & $17.4 \%$ & $51.6 \%$ & $15.0 \%$ & $8.0 \%$ & $100.0 \%$ \\
\hline
\end{tabular}

$\chi^{2}(8)=13.898, p=0.080$

\section{DISCUSSION}

The present study investigated the anxiety levels, work violence and self-esteem in a sample of 213 nursing staff members in five major hospitals and eleven Health Centers in the prefecture of Crete.

In the present study, they have been exposed to at least one mobbing behavior in the last 12 months and at least once a week, almost daily or daily the $11.3 \%$ of the total sample $(\mathrm{n}=24)$, and these people, according to Leymann are the victims of mobbing [26]. In a relevant study carried out in Cyprus in 2014 and the study sample was made up of 136 health professionals (44 general practitioners, 50 nurses, 42 other health professionals) working in Primary Health Care in Nicosia and was used the same questionnaire of the Leymann Inventory of Psychological Terrorization (LIPT) as used in the present study, the prevalence of "mobbing syndrome'among health professionals according to Leymann's official definition was $8.8 \%$ of the total sample reported exposure at least one behavioral psychological violence in the last 12 months, while $11 \%$ reported exposure to at least one "mobbing behaviour'at least once a week. In another study when the Leymann definition was applied, 5.9\% of the participants reported exposure to at least one bullying behavior at least once a week and for at least 6 months [27].

The analysis of the data from the present study showed that the most common "mobbing'behaviors reported among the participants were: "they speak badly for you behind your back'and the same percentage follows "they interrupt you while you express'15.0\%), "receive scornful eyes and / or scornful gestures'(14.6\%), "spread false rumors about you", and with the same percentage they appear to "revile you and shout' $(12.2 \%)$ and finally, they 'they are constantly criticizing your work'(11.3\%). According to Hira et al. (2014), the most common mobbing behaviors reported were the following: 'being continuously interrupted'(17.2\%), "continuously being given new work assignments'(13.5\%), "being gossiped'(11.8\%), "being exposed to slanders and lies'(10.5\%), and “being criticized regarding work assignments'(9.5\%).

In the present study, the less frequent "mobbing behaviors'identified among nursing staff were "sexual assault' $(0.0 \%)$, "they are only addressed to you in writing' $(0.5 \%)$, and 'they are causing you damage by trying to hurts you financially' $(0.5 \%)$. On the other hand, with the study of Hira et al. (2014), the less frequent "mobbing behaviors'identified among health professionals in Primary Health Care was: "you've been sexually attacked,' "psychological violence,"'physical violence,"'oral or sexual suggestions,'"want to force you to undergo a psychiatric examination "and'accept written threats " $(0 \%$ for all of the statements above $)$ [28].

The $63.5 \%$ of the nurses who participated in the survey stated that they had received psychological violence by a "colleague'(this is why the total figure exceeds $100 \%$ ), which accounts for $43.9 \%$ of all the answers and with the same percentage of "people with a higher hierarchical position or superior". Also, with regard to the gender that commit psychological violence, the $43.2 \%$ of the nurses answered that were "women', with the 
same percentage being "both men and women'and the median price of the number of people who commit psychological violence during this period was $2(\mathrm{IQR}=2)$. According to Zachariadou et al (2015) states that senior officials were identified as those who practiced psychological violence (57.5\%). Health professionals working in public hospitals and Primary Health Care reported having faced hostile attitudes mainly by their superiors (58.3\% vs. 55.9\%, respectively). As for the sex of perpetrators, women were most often identified as those who had commit psychological violence compared to men, but there was no statistical significance (69.1\% vs. 19.8\% p = 0.147) [29] . Another research in Turkey by Picakciefe et al. (2012) to people working in the Primary Care, $70.3 \%$ of the victims were superiors [30]. In the present study the participants stated that they discussed the problems they faced in their workplace related with violence and $68.3 \%$ of the respondents said that among other things they had spoken with their colleague. Also, only 2 of respondents said they did not have a person they could contact but they would like to have, while 3 people said they did not have a person to contact or they did not need. In a study of Zachariadou et al. (2015) it is reported that health professionals working at major public hospitals in Cyprus have discussed their exposure to mobbing behavior at their workplace with a colleague of $67.7 \%$, while the $30,6 \%$ with a member of his family [31]..

By applying the definition "Psychological violence at work can be defined as the situation in which an individual is subjected to hostile attitudes by one or more individuals in his working environment which in a continuous and repeated manner seek to destroy him, to oppress him, to abuse him or even to exclude or isolate them for a long time ". 36 nurses (16.9\%) said they have been subjected to psychological violence in their work in the last 12 months. This behavior was attributed to "competition problems between individuals'(60.9\%) and “jealousy'(58.7\%). According to the Hira's study (2014), 30 workers (26.3\%) stated that they had been subjected to psychological violence in the last year according to this definition and this behavior was attributed to "poor organization of work'(43.3\%) followed by "problems in administration'(40\%) [32]. According to Zachariadou et al (2015), 29.3\% of respondents responded positively to exposure to hostile behavior over the last 12 months according to the definition (31.4\% of those working in the large public hospitals in Cyprus and $26.3 \%$ of those working in Primary Health Care respectively). The most common causes of exposure to hostile attitudes towards health professionals working at major public hospitals in Cyprus were «management / placement problems at work 33.3\%' followed by "generally poor working atmosphere 31.3\%," "poor organization $29.2 \%$ 'and «jealousy 29.2\%». In Primary Health Care, the most common causes were «poor organization $43.3 \%$ «, followed by «problems in the administration / placement at work 40\%'and 'poor working atmosphere 33.3\%'and with the same percentage «competition problems among individuals' [33].

To the question: «You have perceived psychological violence against someone else in your workplace in the last 12 months,'30.0\% of nursing staff in the sample of this survey reported that they have perceived such a situation. A similar study reported 38 workers (31.7\%) who responded positively to the question [34]. In another survey, respondents reported that they had notice (43.4\%) bullying behavior against another employee in the previous year. Health professionals working in hospitals in Cyprus noticed that one or more of their colleagues were harassed more frequently than primary care workers in Cyprus (52.2\% vs. $31.4 \%)$, which was statistically significant ( $p=0.001$ ) [35]. In England, Quine (1996), a health care practitioner, it was found that $42 \%$ of the participants had witnessed intimidation behaviors against their colleagues [36].

From the analysis of the results of the STAI questionnaire it was found that the mean value of the trait anxiety was 40.82 while the mean value of the state anxiety was 39.03 and the mean value of the total anxiety was 79.85. A study carried out in 2010 in nursing personeel in the sixth Healthcare Region of Greece working in nephrologic centers showed that trait anxiety was 41.4 and state anxiety 38.6 [37]. Finally, in a research that investigated the trait and state anxiety in nursing personeel working at Thessaly Health Centers, they showed high rates of trait anxiety, which exceeded the corresponding rates for the Greek population [38].

In the present study, the results of the Battle Questionnaire for Self-Esteem showed that $51.6 \%$ of the nursing staff involved in the study had a middle self-esteem, while the lowest percentage of the total sample (8.0\%) 
had a very high self-esteem. Researchers conducted a study in Wales on mental health community nurses who found that most nurses had middle self-esteem (Fothergill et al., 2000) [39]. According to Randle (2003), the result of continuous exposure to bullying includes the reduced of self-esteem [40].

\section{CONCLUSION}

The evaluation of the results shows that the nursing staff of the overall sample of the study experiences mild anxiety symptoms, which necessitate the training of nursing staff in symptom management to avoid increasing their intensity. Study findings indicate that mobbing seems to be at high rates. Prevalence of mobbing is an existing reality in the health sector of Crete. Early recognition of the phenomenon and its management as well as enhancing of the self-esteem should be the best practice of intervention to prevent it.

\section{AREAS OF FurTHER RESEARCH}

Further research is needed to establish workplace violence, anxiety and reduced self-esteem in nursing staff and to establish the reasons for increased odds of work violence and anxiety. Further research is needed to evaluate interventions that minimize the phenomenon of mobbing, anxiety and strengthen of self esteem for example a comparison of the two different kinds of interventions such as group psychotherapy and individual psychotherapy.

\section{REFERENCES}

1. Davenport, N., Schwartz, R.D. \& Elliott, G.P. (1999) Mobbing: Emotional Abuse in the American Workplace. Civil Society Publishing, Ames, IA.

2. Leymann H. Mobbing and psychological terror at workplaces. Violence Vict 1990; 5:119e26.

3. Einarsen, Ståle, Helge Hoel, Dieter Zapf, Carly L. Cooper, Bullying and Emotional Abuse in the Workplace: International Perspectives in Research and Practice, CRC Pres, 2003.

4. Einarsen, S. Harassment and bullying at work; A review of the Scandinavian approach Aggression and Violent Behavior, 5 (4) (2000), pp. 379-401

5. Zapf, D. (1999). Organisational, Work Group Related and Personal Causes of Mobbing/bullying at Work. International Journal of Manpower, 20(1/2), 70-85.

6. H. Hoel, C. CooperDestructive conflict and bullying at work Manchester School of Management. University of Manchester Institute of Science and Technology, Manchester (2000)

7. M. VartiaThe sources of bullying: psychological work environment and organizational climate Eur J Work Organ Psychol, 5 (1996), pp. 203-214

8. K. Niedl Mobbing and well-being: economic and personnel development implications Eur J Work Organ Psychol, 5 (1996), pp. 239-249

9. Zachariadou, T., Zannetos, S., Chira, S., Gregoriou, S., Pavlakis, A. Prevalence and Forms of Workplace Bullying Among Health-care Professionals in Cyprus: Greek Version of "Leymann Inventory of Psychological Terror'Instrument. Safety and Health at Work. 2017 1-8 (Article in press)

10. Siousioura, D. (2010). The Effect of Group Psycho-Educational Intervention on Patients with Type I Diabetes Dissertation. Rhodes: University of the Aegean

11. Papadopoulou, D. (2009). Violence in the Workplace of Nursing Staff. Nursing, 48 (4): 379- 387

12. Panagiotis Koutrouvides (2013). Ministry of Education and Religious Affairs. General .Secretariat for Lifelong Learning. Time management. Educational material for Lifelong . Learning Centers

13. Tsiama M.H., (2013) The phenomenon of moral / psychological harassment in the workplace: conceptual determinations. National Labor and Human Resources Foundation. 
Workplace Violence, Anxiety and Self-Esteem in Nursing Staff of Primary, Emergency and Intensive Care Units on the Island of Crete

14. Spielberger, C.D. (2010). State Trait Anxiety Inventory. Retrivied on 26/04/2017 from:.https://onlinelibrary. wiley.com/doi/pdf/10.1002/9780470479216.corpsy0943

15. Siouisoura, D. (2010). The Effect of Group Psycho-Educational Intervention on Patients with Type I Diabetes Mellitus. Rhodes: University of the Aegean.

16. Mitrousi, S. (2014). Effect of anxiety on the physical and mental health of nurses. Doctoral thesis. Sparta

17. Staurianopoulou Th, Stamati Sta, Gevreki E, Gourvelou, Ou, Papadimitriou M. Level of illness, fatigue, satisfaction and self-esteem of nursing staff in public hospitals in Ilia. Rostrum of Asclepius 10th Volume, $1^{\text {st }}$ Issue, January - March 2011

18. Leymann H. The content and development of mobbing at work. Eur J Work Org Psychol 1996;2:165e84.

19. Niedhammer I, David S, Degioanni S, Drummond A, Philip P. Workplace bullying and sleep disturbances: findings from a large scale cross-sectional survey in the French working population. Sleep 2009; 32: $1211 \mathrm{e} 9$.

20. Spielberger, Charles D.; Sydeman, Sumner J. (1994). "State-Trait Anxiety Inventory and State-Trait Anger Expression Inventory". In Maruish, Mark Edward. The use of psychological testing for treatment planning and outcome assessment. Hillsdale, NJ: Lawrence Erlbaum Associates. pp. 292-321. ISBN 978-0-80581162-9.

21. Niedhammer, I., David, S., Degioanni, S., and et 143 Mıdecins du travail. La version frannaise du questionnaire de Leymann sur la violence psychologique au travail: Le “Leymann Inventory of Psychological Terror'(LIPT). Rev Epidemiol Santı Publique. 2006; 54: 245-262

22. Zachariadou, T., Zannetos, S., Chira, S., Gregoriou, S., Pavlakis, A. Prevalence and Forms of Workplace Bullying Among Health-care Professionals in Cyprus: Greek Version of "Leymann Inventory of Psychological Terror'Instrument. Safety and Health at Work. 2017 1-8 (Article in press)

23. Niedhammer I, David S, Degioanni S, Drummond A, Philip P. Workplace bullying and sleep disturbances: findings from a large scale cross-sectional survey in the French working population. Sleep 2009; 32: $1211 \mathrm{e} 9$.

24. Spielberger, Charles D.; Sydeman, Sumner J. (1994). "State-Trait Anxiety Inventory and State-Trait Anger Expression Inventory". In Maruish, Mark Edward. The use of psychological testing for treatment planning and outcome assessment. Hillsdale, NJ: Lawrence Erlbaum Associates. pp. 292-321. ISBN 978-0-80581162-9.

25. Stalikas, A., Triliva, S. Rousi, P. (2002) Psychometric Instruments in Greece, Greek Letters, Athens, p. 240241

26. Niedhammer, I., David, S., Degioanni, S., and et 143 Mıdecins du travail. La version frannaise du questionnaire de Leymann sur la violence psychologique au travail: Le “Leymann Inventory of Psychological Terror'(LIPT). Rev Epidemiol Santı Publique. 2006; 54: 245-262

27. Zachariadou, T., Zannetos, S., Chira, S., Gregoriou, S., Pavlakis, A. Prevalence and Forms of Workplace Bullying Among Health-care Professionals in Cyprus: Greek Version of "Leymann Inventory of Psychological Terror'Instrument. Safety and Health at Work. 2017 1-8 (Article in press)

28. Chira S. Prevalence of mobbing syndrome between healthcare professionals working at the Primary Healthcare setting of Cyprus. Postgraduate thesis. Open University of Cyprus, Department of Economics and Management. 26/ 08/2014., https://kypseli.ouc.ac.cy/bitstream/handle/11128/1671/\%CE\%94\%CE \%9C\%CE\%A5-2014-00236.pdf?sequence $1 / 41$. 
Workplace Violence, Anxiety and Self-Esteem in Nursing Staff of Primary, Emergency and Intensive Care Units on the Island of Crete

29. Zachariadou, T., Zannetos, S., Chira, S., Gregoriou, S., Pavlakis, A. Prevalence and Forms of Workplace 29. Bullying Among Health-care Professionals in Cyprus: Greek Version of "Leymann Inventory of Psychological Terror'Instrument. Safety and Health at Work. 2017 1-8 (Article in press).

30. Picakciefe M , Acar G, Colak Z, Kilic I. The Relationship between socio-demographic characteristics, work conditions and level "mobbing'of health workers in primary health care. 30thInternational Congress on Occupational Health March 18-23 2012.

31. Zachariadou, T., Zannetos, S., Chira, S., Gregoriou, S., Pavlakis, A. Prevalence and Forms of Workplace Bullying Among Health-care Professionals in Cyprus: Greek Version of "Leymann Inventory of Psychological Terror'Instrument. Safety and Health at Work. 2017 1-8 (Article in press

32. Chira S. Prevalence of mobbing syndrome between healthcare professionals working at the Primary Healthcare setting of Cyprus. Postgraduate thesis. Open University of Cyprus, Department of Economics and Management. 26/ 08/2014., https://kypseli.ouc.ac.cy/bitstream/handle/11128/1671/\%CE\%94\%CE \%9C\%CE\%A5-2014-00236.pdf?sequence $1 / 41$.

33. Zachariadou, T., Zannetos, S., Chira, S., Gregoriou, S., Pavlakis, A. Prevalence and Forms of Workplace Bullying Among Health-care Professionals in Cyprus: Greek Version of "Leymann Inventory of Psychological Terror'Instrument. Safety and Health at Work. 2017 1-8 (Article in press

34. Chira S. Prevalence of mobbing syndrome between healthcare professionals working at the Primary Healthcare setting of Cyprus. Postgraduate thesis. Open University of Cyprus, Department of Economics and Management. 26/ 08/2014., https://kypseli.ouc.ac.cy/bitstream/handle/11128/1671/\%CE\%94\%CE \%9C\%CE\%A5-2014-00236.pdf?sequence $1 / 41$.

35. Zachariadou, T., Zannetos, S., Chira, S., Gregoriou, S., Pavlakis, A. Prevalence and Forms of Workplace Bullying Among Health-care Professionals in Cyprus: Greek Version of "Leymann Inventory of Psychological Terror'Instrument. Safety and Health at Work. 2017 1-8 (Article in press

36. Quine L. Workplace bullying in NHS community trust: staff questionnaire survey. BMJ 1999; 318:228-232.

37. Marneras ch, Theodorakopouloy G, Albani E, Gouva M, Dimopoulou E, Kotrotsiou E. Satisfaction from Work and Anxiety Level in Nurses working in Kidney Centers NOSILEFTIKI 2010, 49 (1): 83-90

38. Faki, M., Gouva, M., Papathanasiou, I., Prapas, P., Pasxou, D., Prapa, E. \& Kotrotsioy, E. (2009). Resistance to Workers in Primary Health Care Structures. Interscientific Health Care, 1, 9-15.

39. Fothergill, A. , Edwards, D. , Hannigan, B. , Burnard, P. and Coyle, D. (2000), Self esteem in community mental health nurses: findings from the all Wales stress study. Journal of Psychiatric and Mental Health Nursing, 7: 315-321.

40. Randle J. 2003. Bullying in the nursing profession. Journal of Advanced Nursing 43: 395-401.

Citation: Maria Maniou, Sofia Zyga, Spyros Vliamos, Panagiotis Prezerakos, Katerina Flora, Andreas Pavlakis, "Workplace Violence, Anxiety and Self-Esteem in Nursing Staff of Primary, Emergency and Intensive Care Units on the Island of Crete". American Research Journal of Nursing. 2018; 4(1): 1-15.

Copyright (c) 2018 Maria Maniou, Sofia Zyga, Spyros Vliamos, Panagiotis Prezerakos, Katerina Flora, Andreas Pavlakis, This is an open access article distributed under the Creative Commons Attribution License, which permits unrestricted use, distribution, and reproduction in any medium, provided the original work is properly cited. 